\title{
Implantação de sistemas informatizados na saúde: uma revisão sistemática
}

\author{
Implementation of health information systems: a systematic review \\ La implementación de los sistemas de salud informatizados: una revisión \\ sistemática
}

Tamyres Oliveira dos Santos | tamyres.enf@gmail.com

Universidade Federal do Rio Grande do Sul, Escola de Enfermagem, Grupo de Estudos em Sistemas Informação e Telessaúde. Porto Alegre, Brasil.

Letícia Passos Pereira | leti passos@msn.com

Universidade Federal do Rio Grande do Sul, Escola de Enfermagem, Grupo de Estudos em Sistemas Informação e Telessaúde. Porto Alegre, Brasil.

Denise Tolfo Silveira | dtolfo@enf.ufrgs.br

Universidade Federal do Rio Grande do Sul, Escola de Enfermagem, Grupo de Estudos em Sistemas Informação e Telessaúde. Porto Alegre, Brasil.

\section{Resumo}

Este estudo, por meio de uma revisão integrativa, analisou as evidências sobre os sistemas de informação e a utilização dos dados para a gestão do cuidado pelos profissionais de saúde. Foram identificados 17 estudos, no período de 2000 a 2013, nas bases Lilacs, SciELO e Medline. Três autores (17,6\%) utilizaram os dados de sistemas para embasar a decisão clínica, seis (35,3\%) para gestão, monitoramento de desempenho de serviços e planejamento de unidades de saúde. Outros seis autores $(35,3 \%)$ definiram que tiveram dificuldades de usar os dados, por falta de terminologia padrão, falhas no processo que resultavam em dados inconsistentes, ou a não identificação por parte dos profissionais com aqueles indicadores em sua realidade. Dois estudos $(11,8 \%)$ relataram suas experiências em informatização. Conclui-se que a área de tecnologia da informação ainda precisa de mais pesquisa, no que diz respeito à mudança de processos e experiências de informatização.

Palavras-chave: enfermagem; Informática em saúde; saúde pública; sistemas de Informação em saúde; indicadores de saúde. 


\begin{abstract}
This study, through an integrative review, analyzed the evidence of the information systems and the use of data for the management of care by health professionals. 17 studies were identified, from 2000 to 2013 , on the databases Lilacs, SciELO and Medline. Three authors (17.6\%) used the system data to support the clinical decision, six authors (35.3\%) for management, monitoring services and planning of community health centers. Six authors (35.3\%) pointed out difficulties to use the datadue to the lack of standard terminology, process failures resulting in inconsistent data, or the non-identification by professionals with those indicators in their reality. Two studies (11.8\%) reported their experiences in informatization. It is concluded that the information technology still needs more research about the change of processes and informatization experiences.
\end{abstract}

Keywords: nursing; health computing; public health; health information systems; health status indicators.

\title{
Resumen
}

Este estúdio analizó la evidencia de los sistemas de información y del uso de datos para la gestión de la atención por los profesionales de salud. Se identificaron 17 estudios, de 2000 a 2013, en las bases Lilacs, SciELO y Medline. Tres autores (17,6\%) utilizaron los datos del sistema para apoyar la toma de decisiones clínicas, seis autores $(35,3 \%)$ para la gestión, los servicios de supervisión del desepeño de servicios y la planificación de zonas básicas de salud. Seis autores (35,3\%) habían declarado dificultades en el uso de datos por falta de terminología estándar, por fallas en el proceso que resultaban en datos inconsistentes, o la falta de identificación entre la realidad de los profesionales y los indicadores. Dos estudios $(11,8 \%)$ relataron sus experiencias con la informatización. Se concluye que la tecnología de la información necesita más investigación sobre la mudanza de los procesos y las experiencias de informatización.

Palabras Clave: enfermería; informática en salud; salud pública; sistemas de información en salud; indicadores de salud.

\begin{abstract}
Contribuição dos autores:
Concepção e desenho do estudo - Tamyres Oliveira dos Santos

Aquisição, análise ou interpretação dos dados - Tamyres Oliveira dos Santos

Redação do manuscrito - Tamyres Oliveira dos Santos; Letícia Passos Pereira

Revisão crítica do conteúdo intelectual - Denise Tolfo Silveira
\end{abstract}

Declaração de conflito de interesses: Não há.

Fontes de financiamento: Não houve.

Considerações éticas: Este estudo foi aprovado pela Comissão de Pesquisa da Escola de Enfermagem e Comitê de Ética e Pesquisa da Universidade Federal do Rio Grande do Sul sob o número 25.437

Agradecimento/Contribuições adicionais: Não há.

Histórico do artigo: Submetido: 28.dez.2015 | Aceito: 11.maio.2017 | Publicado: 29.set.2017

Apresentação anterior: Extraído do trabalho de conclusão de graduação da primeira autora.

Licença CC BY-NC atribuição não comercial. Com essa licença é permitido acessar, baixar (download), copiar, imprimir, compartilhar, reutilizar e distribuir os artigos, desde que para uso não comercial e com a citação da fonte, conferindo os devidos créditos de autoria e menção à Reciis. Nesses casos, nenhuma permissão é necessária por parte dos autores ou dos editores. 


\section{Introdução}

Um dos maiores problemas e, talvez o maior dificultador para o acesso ao atendimento em saúde, é a grande demanda de trabalho burocrático, que não se mostra efetivo e eficiente, a partir do momento em que a 'papelada' se torna uma barreira para o trabalhador, que possui muitas guias para preencher, além do acontecimento de erros, falta de informações, erro de interpretações, entre outras dificuldades. Estes empecilhos, apontados por trabalhadores da saúde e usuários, evidenciam a desorganização do fluxo de informações e do processo de trabalho, aumentando a morosidade no atendimento, limitando cada vez mais o acesso aos usuários do Sistema Único de Saúde (SUS).

A informação em saúde consiste em identificar características individuais e coletivas da saúde de uma população. Os resultados destes dados coletados subsidiam as decisões do gestor, para melhoria da condição de vida ${ }^{2}$. Os Sistemas de Informação em Saúde (SIS) são instrumentos usados para processar os dados e produzir a informação. Podem ser entendidos como instrumentos para adquirir, organizar e analisar dados necessários à definição de problemas e riscos para a saúde, avaliar a eficácia, eficiência e influência que os serviços prestados possam ter no estado de saúde da população, além de contribuirem para a produção de conhecimento acerca da saúde e dos assuntos a ela ligados². Segundo a Organização Mundial de Saúde (OMS), Sistema de Informação em Saúde é um mecanismo de coleta, processamento, análise e transmissão da informação necessária para se organizar e operar os serviços de saúde e, também, para a investigação e o planejamento com vistas ao controle de doenças ${ }^{3}$.

Partindo da ideia de que a informação em saúde possui uma dimensão estratégica, o aparato que envolve essas informações necessita estar a serviço de gestores que, por sua vez, precisam possuir pleno conhecimento do SUS, legislação, realidade epidemiológica, assistencial, financeira, etc., para acompanhar, avaliar e talvez modificar o sistema de informação e, consequentemente, as principais decisões para melhora de saúde da população pela qual é responsável ${ }^{4}$. Além disso, os SIS contribuem para a construção do saber, pois as informações, quando disseminadas, podem desenvolver o conhecimento, não só acadêmico, mas também popular, a partir do momento que podem sumarizar os dados de saúde da população e resultados de ações e programas de saúde. Assim, evidencia-se a necessidade de desenvolver sistemas disponíveis para acesso e uso ao público, com ferramentas que possam contribuir para otimizar a gestão assistencial prestada pelo Sistema Único de Saúde.

Com a regulamentação do SUS, pela Lei no 8.080, os SIS foram valorizados, atribuindo à União, estados e Distrito Federal a organização e coordenação do sistema de informação em saúde. A lei também previa um sistema nacional de informações em saúde, integrado em todo território nacional, abrangendo questões epidemiológicas e de prestação de serviços, organizado pelo Ministério da Saúde em articulação com os níveis estaduais e municipais do SUS.

Além dos grandes bancos de dados gerados por outros setores, o Ministério da Saúde possui SIS com vários subsistemas, que se destacam: SIM (Sistema de Informação de Mortalidade), Sinasc (Sistema de Informações sobre Nascidos Vivos), Sinan (Sistema de Informações de Agravos Notificáveis), Sisvan (Sistema de Vigilância Alimentar e Nutricional), entre outros.

Apesar da quantidade de informações desses subsistemas nacionais e das potencialidades envolvidas, observa-se que eles são subutilizados para os fins a que se destinam frente à natureza do processo de trabalho e do modelo de assistência. Entre as razões, entre outras, temos: a baixa qualidade do preenchimento das informações, a grande quantidade de formulários para um só sistema, a falta de conhecimento da importância desses sistemas por parte dos trabalhadores, a precariedade dos serviços de saúde em estrutura (de tecnologia de informação - TI) e de pessoal (culminando na procrastinação de preenchimento de dados).

Mesmo com toda a evolução da área de TI, existe uma defasagem entre esse avanço e a sua incorporação no processo de gestão em saúde no país. A dificuldade de acesso e do tratamento dos dados existentes, 
de forma rotineira, com disponibilização das informações adequadas no momento necessário, ainda é comum, bem como, a falta de articulação entre os Sistemas de Informação e os processos de planejamento e gestão da saúde ${ }^{5}$.

Sendo o enfermeiro um profissional que exerce funções em todas as esferas do atendimento em saúde - desde o cuidado direto, em todos os níveis de atenção, até a gestão em saúde - ele deve estar atualizado e aberto às modificações pertinentes ao seu processo de trabalho, para melhoria e qualificação do cuidado ao paciente. O que inclui: a) obter melhor entendimento dos papéis e domínios de TI junto à equipe de saúde; b) desenvolver a capacidade de utilizar uma arquitetura de TI para bom uso do sistema, gerenciamento da mudança do processo de trabalho, comunicação e avaliação dos mesmos ${ }^{6}$. Na era da informação, os sistemas informatizados tornam-se uma ferramenta de trabalho indispensável ao profissional de saúde, não só em relação à assistência, mas também em relação à gestão do cuidado.

A partir desse contexto, este estudo teve como objetivo analisar as evidências sobre os sistemas de informação e a utilização dos dados para a gestão do cuidado pelos profissionais de saúde.

\section{Método}

Tratou-se de uma revisão sistemática na qual foram utilizadas as cinco etapas (formulação do problema, coleta de dados, avaliação dos dados, análise e interpretação dos dados e apresentação dos resultados) propostas no método de Cooper. Este método caracteriza-se pelo aspecto de o pesquisador ter interesse de sumarizar resultados de um conjunto de pesquisas sobre um mesmo tema, visando estabelecer generalizações ou desenvolver explicações mais abrangentes de um fenômeno específico a partir da síntese ou análise dos achados dos estudos com propósitos teóricos e ou intervencionistas?

A primeira etapa consistiu na formulação do problema: como são utilizados os dados processados nos sistemas de informação pelos profissionais de saúde? Quais os pontos críticos e os pontos de sucesso na implantação dos sistemas de informação em saúde?

Na segunda etapa ocorreu a coleta de dados, realizada em julho de 2013, nas bases de dados Literatura Latino-Americana e do Caribe em Ciências da Saúde (Lilacs), Scientific Eletronic Library Online (SciELO) e Medical Literature Analysis and Retrieval System OnLine (Medline). Os descritores na busca bibliográfica foram: enfermagem, informática em saúde, saúde pública, avaliação em saúde, sistemas de informação em saúde, indicadores de saúde de acordo com o DeCs (Descritores em Ciências da Saúde). Foram realizados 16 cruzamentos (15 entre dois descritores e um entre três descritores), com os operadores booleanos and.

A terceira etapa compreendeu a avaliação dos dados, conforme as perguntas de pesquisa, os critérios de inclusão e exclusão. Dos 843 documentos encontrados, dezessete artigos compuseram a amostra. Para se chegar à amostra foram excluídos os artigos que tivessem sido publicados até 2003 ( $\mathrm{n}=433)$, que não estivessem disponíveis integralmente online $(\mathrm{n}=84)$, aqueles que não abordavam o contexto estudado $(\mathrm{n}=252)$, além de teses, dissertações e trabalhos de conclusão de curso $(\mathrm{n}=57)$.

Para a quarta etapa, análise e interpretação dos dados, os artigos selecionados foram registrados na forma de quadro sinóptico geral, comparados entre si, buscando-se critérios de semelhança ou diferença entre os mesmos, que serão descritos nos resultados deste estudo.

\section{Resultados}

Os documentos selecionados foram desenvolvidos no Brasil (11), nos Estados Unidos (3) e na Europa (3). Foram escritos por: médicos (5), enfermeiros (8), equipes de estudo multidisciplinar (4). Por tipos de estudos, eles se dividem em: Pesquisa avaliativa (3), Estudo descritivo (7), Revisão bibliográfica (2), Projeto de desenvolvimento (2), Artigo de reflexão (1), Estudo ecológico (1), Série histórica (1). 
Fizeram parte da amostra, ao todo, 17 artigos, com predomínio da língua portuguesa (11 estudos) e do Brasil como país de origem dos periódicos, contrariando o senso comum. Além disso, constatou-se que $58,8 \%$ dos artigos tiveram como metodologia o tipo de estudo descritivo exploratório com abordagem quantitativa predominante, evidenciando o caráter quantitativo dos indicadores disponíveis pelos sistemas de informação.

Após a avaliação inicial dos artigos, os mesmos foram comparados entre si, buscando-se critérios de semelhança ou diferença entre os mesmos. Para organização de ideias e qualificar a discussão, os artigos foram relacionados em três categorias: a) estudos que descrevem como utilizaram os dados de sistemas informatizados; b) estudos que apontaram dificuldade para utilização dos dados de sistemas informatizados e c) pontos críticos e de sucesso na implementação de sistemas informatizados. É importante ressaltar que não houve distinção do tipo de sistema, se privado ou público. Na Figura 1 é apresentado o esquema hierarquizado que sintetiza os resultados encontrados.

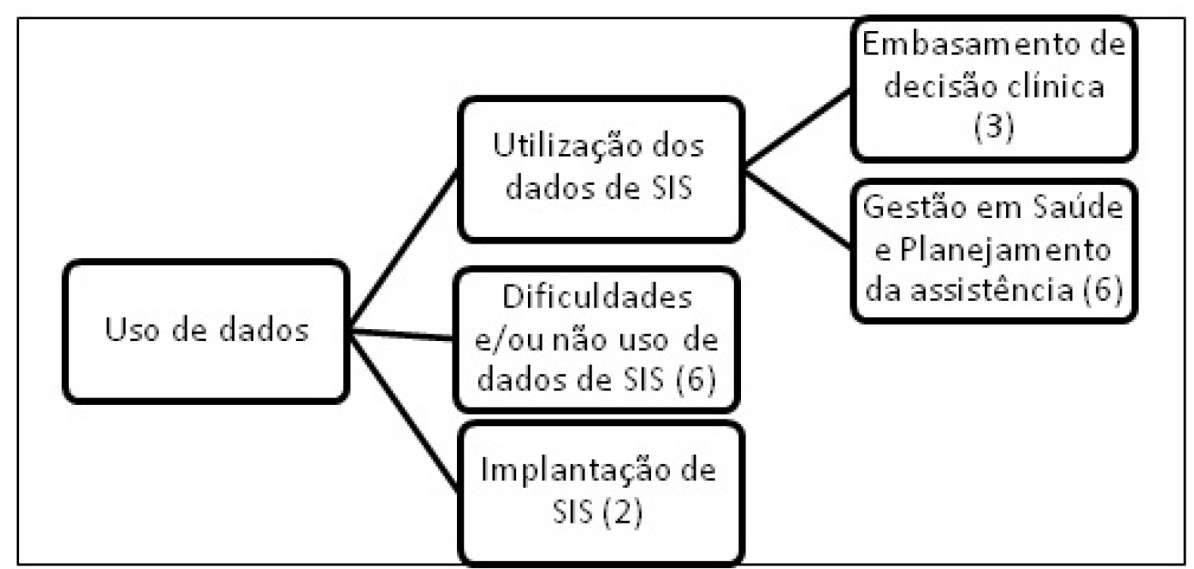

Figura 1 - Esquema-síntese dos resultados encontrados Fonte: Os autores.

Na primeira categoria de análise deste estudo, a qual relacionou os artigos que utilizaram os dados do sistema, observou-se que dos 17 artigos, em 17,6\% (n=3) utilizaram os dados de sistemas informatizados para embasar a decisão clínica, auxiliando na segurança do paciente, envio de dados e monitoramento de adesão a tratamentos. Em 35,2 \% (n=6) dos estudos foram utilizados os dados do sistema para vigilância epidemiológica, gestão dos serviços e sistemas de saúde e planejamento da unidade.

Na segunda categoria de análise estão os estudos $(n=6)$ que não utilizaram os dados ou mostraram dificuldades no uso dos sistemas de saúde. E, na terceira, encontram-se apenas dois estudos que relataram suas experiências de informatização.

\section{Discussão}

Para melhor compreensão e discussão, os resultados foram categorizados em: estudos que utilizaram os dados para embasamento de decisão clínica; estudos que utilizaram os dados para gestão em saúde e planejamento da assistência; dificuldade para utilização dos dados de sistemas informatizados; e pontos críticos e de sucesso na implementação de sistemas informatizados.

\section{Estudos que utilizaram os dados para embasamento de decisão clínica}

A segurança do paciente tem sido um assunto cada vez mais pautado nas instituições de saúde. Pensando nisso, Bates e Gawande ${ }^{8}$ estudaram como a TI pode auxiliar na segurança do paciente, e descobriram que a função mais utilizada e tida como a mais importante pelos profissionais é o monitoramento de 
informações via sistema, que funcionariam como alertas para o suporte de decisões, eventos adversos, interações medicamentosas, riscos aumentados de anafilaxia, entre outros. Outra utilização seria para criação e impressão de pulseiras de diversas cores para riscos como de queda e alergia do paciente, a partir de informações de registros como idade, comorbidades, histórico de doença atual e histórico de quedas ${ }^{8}$. Outro autor relatou que muitos profissionais e especialistas da área da saúde e TI utilizam a funcionalidade de envio e recebimento de imagens por relatórios, como exames, prescrições e planos de cuidado9.

Outro estudo feito por Zhu VJ, Wanzhu TJ e Overhage $\mathrm{M}^{10}$ evidenciam o potencial de dados de sistemas informatizados corretamente alimentados. A equipe de pesquisadores investigou a aderência ao tratamento de diabetes tipo 2 (DM) com o uso de hipoglicemiantes orais e sua relação com a raça/cor, utilizando dados de quantidade de hemoglobina glicada sanguínea pertencentes a um banco de dados, o Rede de Cuidado a Pacientes de Indiana (RCPI - tradução livre), que é um banco utilizado e alimentado há 18 anos por mais de 60 hospitais e clínicas da região de Indianapolis (estado de Indiana - EUA). Os autores concluíram que o uso dos dados do sistema foi essencial para o cruzamento de informações e a avaliação da aderência dos pacientes ao tratamento com hipoglicemiantes. Aqueles pacientes que não apresentavam melhora clínica (diminuição de níveis séricos de hemoglobina glicada) eram contatados e, se necessário, trocava-se ou não o tratamento, abordagem e estratégias ${ }^{10}$.

\section{Estudos que utilizaram os dados para gestão em saúde e planejamento da assistência}

$\mathrm{Na}$ área de gestão e monitoramento do desempenho de serviço de saúde, estão seis estudos. Em um deles, são utilizados os dados do Sistema de Gestão Clínica de Hipertensão Arterial e Diabetes Mellitus da Atenção Básica (Sishiperdia) para investigar se houve aumento no número de clientes portadores de hipertensão arterial sistêmica (HAS) e diabetes cadastrados ${ }^{11}$.

Outro estudo investigou os indicadores de saúde com uma série temporal de 2000 a 2009. Para tanto, utilizaram os dados do Sistema de Informação Ambulatorial (SAI), Sistema de Informação Hospitalar (SIH), Cadastro Nacional de Estabelecimentos de Saúde (CNES), SIM, Sinasc e Sistema de Informação do câncer do colo do útero (Siscolo). Em ambos os estudos se constatou o aumento de cadastrados, e concluiuse que, avaliando e analisando-os sob a perspectiva de expansão da Atenção Primária à Saúde (APS) na época, o aumento do número de equipes de Unidades de Saúde da Família (USF) atuou como catalisador, mostrando indiretamente o quanto a APS é importante como porta de entrada para o paciente do SUS ${ }^{12}$, assim como a informática facilita o trabalho do profissional de saúde e qualifica a assistência ${ }^{13}$.

Outros três estudos utilizam o viés epidemiológico para a gestão e o planejamento das respectivas regiões estudadas. O primeiro discute a situação dos casos de tuberculose (TB) numa região de São Paulo, também numa série temporal de 2000 a 2009, utilizando os dados do Tbweb. Os resultados evidenciaram que as metas para controle da TB não foram alcançadas no que se refere à cura e ao abandono do tratamento ${ }^{14}$. $\mathrm{O}$ segundo utilizou os dados do Sinan para traçar um perfil epidemiológico da hanseníase, e com esses dados detectaram que a grande quantidade de casos de incapacidade sugere o diagnóstico tardio, concluindo-se então que não houve ações eficientes de controle da hanseníase por parte das equipes de atenção básica da região $0^{15}$.

É importante frisar que, quando se trata de doenças crônicas ou de difícil tratamento, os fatores sociais, ambientais, culturais, econômicos são essenciais para a gestão dos programas de saúde. Baseando-se apenas nos dados advindos de sistemas informatizados não se pode avaliar o cuidado prestado e o estado de saúde integral do paciente, já que o contexto dele dificilmente pode ser listado de forma quantitativa a ponto de gerar um número ou um indicador. Sendo assim, é importante que o gestor some informações quantitativas dos sistemas com as questões biopsicossociais para tomar decisões estratégicas para a saúde dessa população. 
O terceiro estudo utiliza um banco de dados de atributos de idosos integrado a um sistema de georreferenciamento para a melhoria de programas de atendimento a idosos. Esta ferramenta se mostrou decisiva para planejamento de ações em saúde voltadas para prevenção de doenças neurológicas e quedas em domicílio, comuns em idosos.

É interessante observar que não se trata apenas de um mapa de riscos e necessidades, relativamente comuns nas USF. Mas sim de um banco de dados contendo atributos (neste caso, dados gerais de identificação, dados do domicílio, família e dados de saúde do paciente idoso) que permitiram a programação de ações voltadas à gerontologia. Este projeto de georreferência amplia possibilidades dos profissionais e gestores de saúde, pois o programa pode ser integrado a banco de dados com outros tipos de atributos, como pacientes hipertensos, diabéticos, obesos, portadores de doenças mentais, tuberculose, entre outros. O fato de ser um banco de dados criado pelo próprio gestor dá a ele possibilidade de modificá-lo conforme suas necessidades, não se tornando algo estanque, como o são muitos sistemas informatizados vendidos no mercado ${ }^{16}$.

\section{Dificuldade para utilização dos dados de sistemas informatizados}

Silveira e Marin ${ }^{17}$ estudaram o uso de conjunto de dados mínimos aplicados à saúde ocupacional e, apesar de reconhecerem o conjunto de dados essenciais como uma ferramenta importante para dar suporte à prática assistencial, reiteram que o uso de alguns sistemas computadorizados demanda uso de terminologias padronizadas diferentes entre si, fazendo com que o enfermeiro pouco explore os sistemas de informação para descrever as suas práticas ${ }^{17}$. Isto reflete também a precariedade com que sistemas de informação são tratados na saúde pública. Na realidade, esses acabam se mostrando, às vezes, como 'mais uma tarefa' e não como uma ferramenta de trabalho, organização e gestão.

Tanto que, ao se discutir quais instrumentos de avaliação de necessidades de saúde são utilizados na USF, descobriu-se que bases de dados como o Sistema de Informação da Atenção Básica (Siab)não são reconhecidas como ferramenta para o reconhecimento de prioridades para a população a que atendem ${ }^{18}$; subentende-se que a programação das ações em saúde é realizada de maneira empírica. Algumas contradições motivadoras para o aparecimento deste problema são: 1) o SUS contém diferentes concepções de necessidades que competem entre si e desencadeiam conflitos na operacionalização; 2) apesar de o SUS ter como princípio a integralidade, operacionaliza as ações por meio de programas específicos (saúde da mulher, criança, homem, idoso), de forma que os profissionais focalizam no processo saúde-doença de cada integrante da família, e não da família como um todo; 3) ainda, neste aspecto, os dados não estão disponíveis por completo online, ou requerem instalação de aplicativos específicos para visualização; 4) os indicadores enfatizam dados de morbimortalidade e patologias, mas pouco relacionam as questões socioeconômico demográficas, geográficas e culturais que interferem no estado de saúde do paciente ${ }^{19,20}$. Outro autor pontua o que, dando continuidade à enumeração: 5) problemas como falhas no preenchimento, 6) equívocos nos dados das Unidades Básicas de Saúde (UBS) e 7) alta rotatividade nos serviços podem ser a justificativa de indicadores imprecisos que não refletem o real trabalho das equipes ${ }^{21}$.

Hanrahan e colaboradores ${ }^{22}$ encabeçaram o desenvolvimento da Rede de Informação de Saúde Pública (Risp - em tradução livre), que é um programa que se propõe a ser um sistema de vigilância em saúde. O objetivo dos criadores é agregar as informações de meio ambiente, saneamento, poluição, trânsito e relacionar com casos de cânceres infantis. Para isso, integraram informações de diversos sistemas, mas notaram que os dados do sistema ainda são limitados, pois não possuíam marcadores de exposição e suscetibilidade individual a poluentes, e concluíram que esse tipo de sistema não suportaria o rastreamento ambiental. Sendo assim, nota-se aqui uma inconsistência: o esforço financeiro e social para a construção de um sistema que se concluiu ser relativamente inválido ao que se propunha naquele momento. 
Estudo com objetivo de estruturar um modelo de relatório visando à utilização em hospitais universitários para a tomada de decisão demonstrou que a coleta não era realizada de forma sistemática, tendo em vista que em geral quem coletava os dados na instituição não sabia da importância que esses dados teriam no final da cadeia, nem das condições de visibilidade sobre a organização do serviço hospitalar, possibilitando assim correções e ações futuras ${ }^{23}$. Sendo o SUS o órgão financiador das internações, a produção de informação dos indicadores na gestão hospitalar, frequentemente mais utilizados pela direção e gerência, pode se tornar uma potencial ferramenta de suporte para prover a instituição de informações necessárias à tomada de decisão, por exemplo.

\section{Pontos críticos e de sucesso na implementação de sistemas informatizados}

A implementação de sistemas informatizados, que engloba diversos esforços de diferentes níveis da sociedade, requer no mínimo planejamento, organização, financiamento, preparação, capacitação e execução, avaliação e planejamento novamente. O primeiro fator a observar é o fato de que apenas dois artigos (11,8\% da amostra total) fazem menção à implementação de sistemas informatizados. Desta forma é complicado fazer muitas inferências, já que o resultado de apenas dois autores pode se tornar tendenciosa. Mas com apenas dois estudos já podemos refletir sobre pontos em comum em ambos. Hyppönen, Ammenwerth, Nohr, Faxvaag e Walldius ${ }^{24}$ avaliaram os indicadores utilizados na implantação de tecnologias de informação na saúde. Ferreira JBB, Mishima SM, Santos JS, Forster AC e Ferraz $\mathrm{CA}^{25} \mathrm{em}$ um estudo pioneiro descrevem a avaliação da instalação de um complexo regulador de consultas especializadas e exames na cidade de Ribeirão Preto. Como pontos de sucesso eles mencionam: 1) Organização do Complexo Regulador (CR); 2) Garantia de acesso e equidade; 3) Constituição da rede de atenção resolutiva e humanizada; 4) Mudança de processo de trabalho dos sujeitos no CR; 5) Uso do CR como estratégia de observador da rede de saúde. A regulação da oferta e a demanda de saúde de uma cidade é um dos pontos essenciais para garantia de acesso, equidade, integralidade e universalidade, seguindo, portanto, os preceitos do SUS.

Apesar de o CR trabalhar como órgão de apoio aos três níveis de atenção em saúde, é importante observar que o complexo regulador reflete como se dão as relações da rede de saúde com os pacientes e dos serviços de saúde entre si. Um exemplo claro é a quantidade de novas solicitações de consultas especializadas, que no caso de Ribeirão se mantiveram estáveis, refletindo o modelo de saúde pautado na especialização médica e não na saúde da família, de prevenção e promoção de saúde.

Como pontos críticos os dois autores concordam que: 1) há necessidade de investimentos, divulgação e aperfeiçoamento; 2) não há critérios para acompanhamento e avaliação de implantação; 3) o uso de diferentes medidas para diferentes projetos dificulta a mensuração do impacto de TI na saúde.

O que presenciamos são projetos isolados, iniciativas inovadoras, motivados por gestores visionários, mas que não refletem um todo dos estados e do país. Sendo assim, a falta de investimentos ou a má utilização de verbas na área da tecnologia da informação é ainda menos pautada, já que esse tipo de tecnologia é inicialmente caro, e o pouco de investimento que se tem é gasto para atividades assistenciais e emergenciais, como consultas, medicações, curativos, equipamentos de saúde, reformas de unidades, etc.

Os gestores em geral sabem que a TI pode e auxilia muito o trabalho, agiliza e o quantifica, mas não pensam nisso como prioridade para suas gestões. Com o aumento das auditorias de contas, da necessidade de cobrança correta aos prestadores dos serviços além de melhora no atendimento, é possível localizar algumas iniciativas de informatização em cidades brasileiras. No entanto, estas são pouco divulgadas no meio científico, limitando-se a notícias de jornais e televisão, nem sempre positivas ao apresentarem os resultados prévios desses projetos.

O que nos leva ao segundo ponto crítico citado pelos autores, que dizem que não há uma padronização de avaliação e acompanhamento desses processos. Sendo assim, o mínimo sinal de aumento de fila em 
uma unidade ou órgão público que passa por essas mudanças de processos já é o bastante para uma capa de jornal/site noticiando a demora ou o transtorno causado pelo sistema. Notícias essas que normalmente focam o sistema como um problema, não questionando ou visando a algo muito maior que é a mudança de processo de trabalho em saúde e gestão organizada e equitativa.

\section{Considerações finais}

Apenas em 17,6\% (n=3) dos artigos o tema central foi a TI na área da saúde, refletindo o fato de que os profissionais de saúde no geral não falam de TI, não só por não ser sua área, mas por ser baixa a adesão e existência de cursos e especializações em relação à TI. Isso é evidenciado pelo fato de apenas dois autores relatarem suas experiências de informatização (respondendo à segunda pergunta). Reiteramos a necessidade dos gestores e academia fomentarem nos profissionais a troca de conhecimentos com relação a TI em saúde, até mesmo por meio de rodas de conversa, e atividades que possam auxiliar os profissionais a conhecer as ferramentas que possuem para tomadas de decisões; do contrário os SIS serão apenas 'mais uma tarefa'. Partindo para uma esfera maior, os gestores também necessitam ler e tomar conhecimento das experiências em outras secretarias municipais, estaduais e no Ministério, e dividir dificuldades e conquistas, para colaboração e até mesmo oportunizar pactuações intergestoras.

Com base neste estudo, pode-se concluir que os dados de sistemas informatizados auxiliam no suporte à decisão clínica, diminuindo a chance de erros e acelerando a tomada de decisão por parte dos profissionais. Apenas ressalva-se que o computador e o sistema devem ser apenas ferramentas que subsidiem o processo de trabalho desse profissional, e não pode ser visto como finalidade, atrapalhando o atendimento em saúde e deixando-o mais distante pela presença de equipamentos de informática.

Assim, para aumentar a utilização dos dados em saúde pelos profissionais, usuários e comunidade acadêmica, torna-se necessário otimizar o acesso aos sistemas de informação existentes. Como as bases de dados convergem para o DATASUS, com indicadores importantes, cabe aos gestores, acompanhar e aconselhar o profissional de saúde que alimentam e utilizam os sistemas implementados, evitando que os SIS funcionem apenas como repositório sem refletir o cuidado prestado em determinado local. Outro ponto é a precariedade com que as vezes são inseridas as informações, e pouco servem para programar ações de saúde dirigidas à população na qual se baseiam os dados do sistema, no caso das USF. Além disso, questiona-se até que ponto os SIS contemplam as reais necessidades dos profissionais assistenciais para a análise, a investigação e a estruturação das ações em saúde.

Sendo assim, acredita-se que com o bom uso e com a alimentação de dados dos sistemas, correta e atualizada, é possível criar relatórios consistentes, que nos darão uma imagem nítida e correta da situação dos clientes que temos, facilitando atividades de monitoramento. A partir dos achados, pôde-se refletir que enquanto o dado for apenas um alerta, uma imagem, um relatório, não será nada mais do que apenas isso. O dado só se transforma em informação quando utilizado pelos profissionais de saúde, e, por conseguinte, o investimento financeiro (englobando a questão econômica de recursos humanos e de infraestrutura) realizado para obter esse tipo de dado também só será aproveitado por ocasião da utilização desse dado para realizar o cuidado do paciente e da população baseado em evidências clínicas e epidemiológicas, transformando-se em informação e, por conseguinte, qualificando a atenção em saúde, os profissionais e melhorando a situação de saúde da população. 


\section{Referências}

1. Fernandes LCL, Machado RZ, Anschau GO. Gerência de serviços de saúde: competências desenvolvidas e dificuldades encontradas na atenção básica. Ciênc Saúde Colet. 2009;14 Suppl 1:1541-52.

2. Branco MAF. Sistemas de informação em saúde no nível local. Cad. Saúde Pública. 1996 jun. 12;(2):267-70.

3. Moraes IHS. Considerações conceituais. In: Moraes IHS. Informações em saúde: da prática fragmentada ao exercício da cidadania. São Paulo: Hucitec; 1993. p.19-38.

4. Silva GFS, Aidar T, Mathias TAF. Qualidade do sistema de informações de nascidos vivos no estado do Paraná, 2000 a 2005. Rev Esc Enferm USP. 2011;45(1):79-86.

5. Bastos MP. Sistemas de informação em saúde: o seu uso no acompanhamento de pacientes hipertensos e diabéticos, um estudo de caso do Sistema Remédio em Casa utilizado no município do Rio de Janeiro [dissertação]. Rio de Janeiro: Escola Nacional de Saúde Pública; 2009.

6. Yasnoff WA, O'Carroll PW, Koo D, Linkins RW, Kilbourne EM. Public health informatics: improving and transforming public health in the information age. J Public Health Manag Pract. 2000 Nov; 6(6):67-75.

7. Cooper HM. The integrative research review. A systematic aproach. Newburg Park: Sage; 1982.

8. Bates DW, Gawande AA. Improving safety with information technology. N Engl J Med. 2003 Jun $19 ; 348(25): 2526-34$.

9. Kern LM, Wilcox A, Shapiro J, Dhopeshwarkar RV, Kaushal R. Which components of health information technology will drive financial value? Am J Manag Care. 2012 Aug; 18(8):438-45.

10. Zhu VJ, Tu W, Marrero DG, Rosenman MB, Overhage JM. Race and medication adherence and glycemic control: findings from an operational health information exchange. AMIA Annu Symp Proc. 2011; 2011:1649-5.

11. Lima ETA, Muniz IG, Santos RF, Bezerra SMMS, Medeiros CCM, França ISX. Correlação entre o número de hipertensos cadastrados no programa hiperdia e a ampliação da estratégia de saúde da família: estudo descritivo. Rev Bras Enferm. 2009; 8(3).

12. Sala A, Mendes JDV. Perfil de indicadores da atenção primária à saúde no estado de São Paulo: retrospectiva de 10 anos. Saúde soc. 2011; 20(4):912-26 .

13. Silva GFS, Aidar T, Mathias TAF. Qualidade do Sistema de Informações de Nascidos Vivos no Estado do Paraná, 2000 a 2005. Rev Esc Enferm USP. 2001; 45(1):79-86.

14. Hino P, Takahashi RF, Bertolozzi MR, Egry EY. A ocorrência da tuberculose em um distrito administrativo do município de São Paulo. Esc. Anna Nery Rev. Enferm. 2013; 17(1):153-9.

15. Lana FCF, Carvalho APM, Davi RFL. Perfil epidemiológico da hanseníase na microrregião de Araçuaí e sua relação com ações de controle. Esc. Anna Nery Rev. Enferm. 2011; 15(1):62-7.

16. Pavarini, SCI, Mendiondo EM, Montaño M, Almeida DMF, Mendiondo MSZ, Barham EJ, et al. Sistema de informações geográficas para a gestão de programas municipais de cuidado a idosos. Texto Contexto Enferm. 2008; 17(1):17-25.

17. Silveira DT, Marin HF. Conjunto de dados mínimos de enfermagem: construindo um modelo em saúde ocupacional. Acta Paul Enferm. 2006; 19(2):218-27.

18. Egry EY, Oliveira MAC, Ciosak SI, Maeda ST, Barrientos DMS, Fonseca, RMGS, et al. Instrumentos de avaliação de necessidades em saúde aplicáveis na estratégia de saúde da família. Rev Esc Enferm USP. 2009; 43(2):1181-6.

19. Santiago AC, Fracolli LA, Zoboli ALCPZ, Silva MV. Indicadores sociais e de saúde para a operacionalização da Vigilância à Saúde. Rev Esc Enferm USP. 2008; 42(4);798-803.

20. Kilpeläinen K, Tuomi-Nikula A, Thelen J, Gissler M, Sihvonem AP, Kramers P, et al. Health indicators in Europe: availability and data needs. Eur J Public Health. 2012 Oct; 22(5):716-21.

21. Malfatti CRM, Assunção A. Hipertensão arterial e diabetes na estratégia de saúde da família: uma análise da frequência de acompanhamento pelas equipes de saúde da família. Ciên Saúde Coletiva. 2011; 16 Suppl 1:1383-8. 
22. Hanrahan LP, Anderson HA, Busby B, Bekkedal M, Sieger T, Stephenson L, et al. Wisconsin's Environmental Public Health Tracking Network: Information Systems Design for Childhood Cancer Surveillance. Environ Health Perspect. 2004 Oct; 112(14):1434-9.

23. Cintra RF, Vieira SFA, Hall, Rosemar J, Fernandes CR. A informação do setor de faturamento como suporte à tomada de decisão: um estudo de caso no Hospital Universitário da UFGD. Ciênc. Saúde Coletiva. 2013 out.; 18(10):3043-53.

24. Hyppönen $\mathrm{H}$, Ammenwerth $\mathrm{E}$, Nohr C, Faxvaag A, Walldius A. eHealth indicators: results of an expert workshop. Stud Health Technol Inform. 2012; 180:328-32.

25. Ferreira JBB, Mishima SM, Santos JS, Forster AC, Ferraz CA. O complexo regulador da assistência à saúde na perspectiva de seus sujeitos operadores. Interface (Botucatu) 2010; 14(33):345-58. 